doi:10.1017/S0022215116005302

\section{ID: IP034}

A new look at middle ear adhesions: transcanal endoscopic approach to aditus block

Presenting Author: Manuela Fina

Manuela Fina

Assistant Professor, University of Minnesota

Learning Objectives: To illustrate the anatomy of the middle ear ventilation routes to the attic, to understand the pathophysiology that leads to aditus block syndrome and to show how to functionally restore the blocked pathways of ventilation.

Introduction: This narrated six minutes surgical video illustrates a novel concept to treat aditus ad antrum block syndrome through an exclusive transcanal endoscopic approach. Granulation tissue and mucosal webs obstructing the pathways of ventilation from the tympanic isthmus to the mastoid space are removed endoscopically with sparing of mastoidectomy and preservation of mastoid mucosa.

Methods: A pre-operative CT scan of the temporal bone was obtained for surgical planning. The surgery was performed entirely through a transcanal endoscopic approach with rigid 0 and 30 degree endoscopes, $3 \mathrm{~mm}$ in diameter and $14 \mathrm{~cm}$ in length, connected to a video camera and high definition monitor. Inflammatory granulation tissue and mucosal webs obstructing the movement of the ossicular chain and the ventilation routes to the mastoid were removed with sharp dissection without mastoidectomy. The routes of ventilation to the mastoid were re-established and irrigated through a transcanal endoscopic limited atticotomy and the small scutal defect was repaired with a cartilage graft.

Results: Inflammatory granulation tissue and mucosal webs obstructing the pathways to mastoid ventilation were removed successfully through a transcanal endoscopic approach with integrity of the mastoid space and preservation of mastoid mucosa.

Conclusion: This endoscopic minimally invasive novel approach to mastoid ad antrum block syndrome focuses on functional restoration of the aeration pathways through the tympanic isthmus instead of removal of disease through mastoidectomy. With this approach the epitympanic and mastoid mucosa is preserved to maintain the important function of mucosal gas exchange, buffer mechanism and homeostasis of middle ear ventilation.

\section{doi:10.1017/S0022215116005314}

\section{ID: IP035}

Endoscopic Combined Approach for Attic Cholesteatoma with Mastoid Extension
Presenting Author: Manuela Fina

Manuela Fina

Assistant Professor, University of Minnesota

Learning Objectives: This eight minutes narrated surgical video demonstrates the surgical principles of the combined endoscopic and microscopic approach for an extensive epitympanic cholesteatoma with mastoid extension.

Introduction: This narrated video lecture illustrates the indications to utilize a combined technique with transcanal endoscopic atticotomy and microscopic mastoidectomy for cholesteatoma with mastoid antrum extension in a pediatric case.

Methods: A pre-operative endoscopic exam and a CT scan of the temporal bone were obtained for surgical planning. The endoscopic portion of the surgery was performed with rigid 0,30 and 45 degree endoscopes, $3 \mathrm{~mm}$ in diameter and $14 \mathrm{~cm}$ in length, connected to a three chip video camera and high definition monitor. A microscopic mastoidectomy was performed to remove disease in the mastoid antrum.

Results: Cholesteatoma was removed in its entirety through a combined endoscopic and microscopic approach. The cholesteatoma extended medially to the ossicular chain and the head of malleus and incus were removed. Ossicular chain reconstruction was performed with autologous incus. The atticotomy defect was reconstructed with a composite graft of conchal cartilage and perichondrium. The patient was free of disease at second look transcanal endoscopic surgery with excellent hearing result.

Conclusions: Endoscopic ear surgery offers wide field visualization of the attic space and the mastoid antrum. When cholesteatoma has extension into the mastoid space posteriorly to the point of reach of the endoscopic technique, a microscopic mastoidectomy is required for complete eradication of mastoid disease. This surgical case demonstrates how the endoscopic and microscopic surgical approach to cholesteatomas are not exclusive of each other but they can be utilized in combination to fully visualize and remove extensive disease.

doi:10.1017/S0022215116005338

\section{ID: IP037}

A case of idiopathic oculostapedial synkinesis without history of facial nerve palsy

Presenting Author: Keishi Fujiwara Keishi Fujiwara ${ }^{1}$, Yasushi Furuta ${ }^{2}$, Shinya Morita ${ }^{1}$, Atsushi Fukuda ${ }^{1}$, Akihiro Homma ${ }^{1}$, Takatsugu Mizumachi ${ }^{1}$, Satoshi Fukuda ${ }^{1}$

${ }^{1}$ Hokkaido University, ${ }^{2}$ Teine-Keijinkai Hospital 


\section{Learning Objectives:}

Objective: To report a case of idiopathic oculostapedial synkinesis without facial nerve disorder.

Patient: A 30-year-old woman with tinnitus synchronous with eye closure is presented. The patient had no history of facial nerve disorder.

Result: An impedance audiometer, in the absence of an auditory stimulus, was used to record tympanic membrane compliance without sound stimulation, revealing decreased compliance in the concomitant with eye blinking. Her symptoms disappeared spontaneously, so no intervention was undertaken.

Conclusion: Although oculostapedial synkinesis is often observed as one of the sequelae of facial nerve palsy, idiopathic oculostapedial synkinesis is very rare. The use of an impedance audiometer in the absence of an auditory stimulus is very useful for demonstrating objective changes in the compliance of the tympanic membrane. It is assumed that the cause of the synkinesis in our case was abnormal transmission of signals for orbicularis oculi muscle to the stapedial and orbicularis oris muscles rather than misdirected regenerating fibers. Resection of the stapedial muscle tendon should be considered if her symptoms recur.

doi:10.1017/S002221511600534X

\section{ID: IP038}

Factors influencing the decision-making of cochlear implantation in congenital

hearing loss: A retrospective cohort study

Presenting Author: Takashi Fujiwara

Takashi Fujiwara

Kurashiki Central Hospital

\section{Learning Objectives:}

Background / Objective: Cochlear implantations (CIs) are well recognized and accepted treatment for severe to profound congenital hearing loss, but CIs are not performed because of malformation, family attitude and others. We conducted retrospective chart review to examine the clinical factors influencing the decision making of CIs in congenital hearing loss.

Study Design: Retrospective chart review.

Methods: We included bilateral congenital hearing loss children who first visited Ehime Welfare Center for the Handicapped from April 2007 to December 2015, and met the criteria of the indication for CsI. To examin factors associated with opt-out cochlear implantation, we performed univariate analyzes of following factors; age, sex, birth weight, maternal age, cochlear malformation, multiple organ abnormalities, severe handicap, deaf family and availability of CIrehabilitation service.
Results: During the study period, 38 bilateral congenital hearing loss children met the criteria for cochlear implantation. Of 38 children, 10 children were unwilling to use CIs. In univariate analysis, severe handicap and deaf family are correrate with opt-out CIs.

doi:10.1017/S0022215116005351

\section{ID: IP039}

Diffusion-weighted MR imaging for

evaluation of cholesteatoma and the value

of $\mathrm{T} 1$ weighted MR imaging in the

exclusion of the false-positive

Presenting Author: Atsushi Fukuda

Atsushi Fukuda, Shinya Morita, Keishi Fujiwara,

Takatsugu Mizumachi, Yuji Nakamaru, Akihiro Homma, Satoshi Fukuda

Hokkaido University Graduate School of

Medicine

Learning Objectives:

Introduction: Magnetic resonance imaging (MRI) is becoming increasingly used as a tool for cholesteatoma diagnosis. The purpose of this retrospective study was to assess a role of T1 weighted imaging (T1WI) in diagnosing recurrent temporal bone cholesteatoma with Diffusion-weighted MR imaging (DWI).

Method: We studied 44 patients (45 temporal bones) with preoperatively suspected cholesteatoma. Each patient underwent an MRI examination including both DWI and T1WI. Diagnosis of cholesteatoma was based on the evidence of a high intense image on DWI. Results of MRI were compared with operative diagnosis.

Result: The patients were consisted of 24 males and 20 females, ranging in age between 8 and 87 (median age $=$ 53). DWI accurately predicted the presence of cholesteatoma in 31 of 36 cases, and it correctly excluded in 5 of 9 cases. False positives included 2 cholesterol granulomas, 1 schwannoma, and 1 fibrosis. False negatives included 4 small keratin pearls, 1 wetter debris caused by infections. Overall sensitivity and specificity for detection of cholesteatoma were $86.1 \%$ and $55.6 \%$, respectively. Positive predictive value and negative predictive value were $88.6 \%$ and $50.0 \%$, respectively. Overall accuracy for detection of cholesteatoma was $80.0 \%$.Only $5.6 \%$ of cholesteatomas $(2 / 36)$ showed high intensity on T1WI, on the other hand, $75.0 \%$ of false positives $(3 / 4)$ showed high intensity on T1WI. When diagnosis of cholesteatoma was based on the evidence of both high intensity on DWI and low or intermediate intensity on T1WI, overall accuracy for detection of cholesteatoma increased to $82.2 \%$.

Conclusion: The combination of DWI and T1WI may improve specificity and overall accuracy for detection of cholesteatoma. 